This article is licensed under the Creative Commons Attribution-NonCommercial 4.0 International License (CC BY-NC) (http://www.karger.com/Services/OpenAccessLicense). Usage and distribution for commercial purposes requires written permission.

\title{
Combination Treatment of Low-Frequency Repetitive Transcranial Magnetic Stimulation and Intensive Occupational Therapy for Ataxic Hemiparesis due to Thalamic Hemorrhage
}

\author{
Naoki Urushidani ${ }^{a} \quad$ Takatsugu Okamoto $^{a, b} \quad$ Shoji Kinoshita ${ }^{a, b}$ \\ Shingo Yamane Hiroaki Tamashiro $^{a}$ Wataru Kakuda ${ }^{d}$ Masahiro Abo ${ }^{b}$ \\ ${ }^{a}$ Nishi-Hiroshima Rehabilitation Hospital, Hiroshima, Japan; ${ }^{b}$ Department of Rehabilitation \\ Medicine, The Jikei University School of Medicine, Tokyo, Japan; 'Institute of Biomedical \\ \& Health Sciences, Hiroshima University, Hiroshima, Japan; ${ }^{d}$ Department of Rehabilitation \\ Medicine, International University of Health and Welfare, Mita Hospital, Tokyo, Japan
}

\section{Keywords}

Ataxic hemiparesis · Thalamus · Repetitive transcranial magnetic stimulation · Occupational therapy $\cdot$ Rehabilitation

\begin{abstract}
Background: Both low-frequency repetitive transcranial magnetic stimulation (LF-rTMS) and intensive occupational therapy (OT) are clinically beneficial for post-stroke patients with upper-limb hemiparesis. However, the usefulness of LF-rTMS and intensive OT for ataxic hemiparesis (AH) is unknown. Methods: The study subjects included 7 patients with AH. All patients had ataxia and mild hemiparesis without a sensory disturbance that was due to thalamic hemorrhage. Each patient was scheduled to receive 20 -min rTMS at $1 \mathrm{~Hz}$ at the contralesional cerebral hemisphere followed by 120 -min intensive OT, daily for 21 sessions. The
\end{abstract}




\section{Case Reports in Neurology}

primary outcome was the motor function of the affected upper limb that was evaluated by using the Fugl-Meyer Assessment (FMA). In addition, the International Cooperative Ataxia Rating Scale (ICARS) score was determined to assess the severity of ataxia. Results: All patients completed the protocol without any adverse effects. The FMA score significantly increased after treatment. Notably, the ICARS score also significantly decreased. Conclusions: Our proposed combination treatment is a safe and feasible neurorehabilitative intervention for patients with $\mathrm{AH}$ due to thalamic hemorrhage. Our results demonstrate the possibility that rTMS in combination with intensive OT could improve motor function and alleviated ataxia in patients with $\mathrm{AH}$.

(C) 2017 The Author(s)

Published by S. Karger AG, Basel

\section{Introduction}

In 1965, Fisher and Cole [1] reported cerebellar ataxia cases that were caused by lesions other than those in the cerebellum, describing a syndrome that consisted of a combination of homolateral ataxia and paresis. The syndrome was later renamed "ataxic hemiparesis (AH)," signifying any combination of weakness and incoordination out of proportion on the same side of the body. This condition can result from a small brain lesion localized at various sites, such as at the pons, midbrain, internal capsule, corona radiata, and cerebral cortex [2]. Subsequent to the report by Fisher and Cole, a few other cases of AH were reported that were caused by a thalamic lesion [3]. Brain imaging studies indicate that thalamic lesions are rarely implicated in $\mathrm{AH}[4]$.

AH following a thalamic lesion is most likely due to an interruption of the dentatorubro-thalamo-cortical fibers at the level of the injured ventrolateral nucleus [3]. Furthermore, nonsensory AH due to a thalamic lesion is usually caused by a lacunar infarction [3], and a few cases are caused by a hemorrhage [5].

Recently, repetitive transcranial magnetic stimulation (rTMS) was introduced as a therapeutic tool for upper-limb hemiparesis after stroke. We reported that a combined application of low-frequency rTMS (LF-rTMS) over the intact hemisphere and the Novel Intervention Using Repetitive TMS and Intensive Occupational Therapy-15 Day (NEURO-15) protocol, which is a constraint-intensive rehabilitation program, significantly improved the motor function of the affected upper limb in post-stroke patients [6]. An electrophysiological study used TMS for AH cases involving a thalamic lesion [7], but there have been no studies of rTMS for rehabilitation in this regard.

In the present study, we firstly applied the NEURO-15 protocol (LF-rTMS and intensive occupational therapy [OT]) in 7 patients with nonsensory AH due to thalamic a hemorrhage to clarify the safety and feasibility of this treatment intervention for upper-limb hemiparesis with ataxia. 


\section{Case Reports in Neurology}

\section{Methods}

Subjects

In total, 177 post-stroke patients with upper-limb hemiparesis were admitted to the $\mathrm{Ni}$ shi-Hiroshima Rehabilitation Hospital to receive our combination protocol of LF-rTMS and intensive OT between October 1, 2010, and September 30, 2015. Among these patients, 14 had hemiparesis and ipsilateral ataxia with a thalamic lesion that was confirmed based on their medical information and a computed tomography (CT) scan. We excluded 7 of these patients because they presented with ataxia due to a disturbance of deep sensibility. Finally, the subjects selected for our analysis included 7 patients who were diagnosed with AH due to a thalamic lesion.

As reported by Moulin et al. [4], the criteria for treatment of AH using the NEURO-15 protocol are as follows: (1) Brunnstrom recovery stage 4-6 for the hands and fingers, and the upper limb of the affected side; (2) a cerebellar type of discoordination that cannot be explained by the severity of the hemiparesis, with dysmetria, defined as poorly controlled direction movement on finger-to-nose or finger-to-finger tests, and dysdiadochokinesia; (3) no sensory deficit; (4) no evidence of a visual field defect; (5) age at intervention 18-80 years; (6) time after onset of stroke of more than 12 months; (7) history of a single symptomatic stroke only (no bilateral cerebrovascular lesion); (8) no cognitive impairment with a pre-treatment Mini-Mental State Examination score of more than 24; (9) no active physical or mental illness requiring medical management; (10) no recent history of seizure; (11) no documented epileptic discharge on a pretreatment electroencephalogram; and (12) no pathological conditions that are contraindications for rTMS in the guidelines suggested by Wassermann [8]. The clinical characteristics of the 7 patients in the present study are shown in Table 1 . The mean age at admission was 67.7 years, and the mean time between the onset of stroke and intervention was 66.6 months. The clinical diagnosis of all patients was thalamic hemorrhage.

During a 15-day hospitalization, each subject received 21 treatment sessions of daily 20min LF-rTMS and 120-min intensive OT (2 sessions per day) (Fig. 1). The motor function of the affected upper limb was serially evaluated on the day of admission and the day before discharge.

\section{Low-Frequency Repetitive Transcranial Magnetic Stimulation}

LF-rTMS was applied using a 70-mm figure 8 coil and MagPro R30 stimulator (MagVenture Company, Farum, Denmark). In each session, rTMS of 1,200 pulses with $1 \mathrm{~Hz}$ was applied to the skull of the nonlesional hemisphere at a site that elicited the largest motorevoked potentials in the first dorsal interosseous muscle of the unaffected upper limb on surface electromyography. The stimulation intensity was set at $90 \%$ of the motor threshold of the first dorsal interosseous muscle, which is the lowest intensity of stimulation that can activate motor-evoked potentials of that muscle. All patients were monitored carefully throughout the rTMS session by the physiatrist applying the rTMS.

\section{Intensive OT}

The 60-min one-on-one training was introduced individually in a face-to-face fashion by an occupational therapist. The main purposes of the training session were to improve the 


\section{Case Reports in Neurology}

upper limb hemiparesis and ataxia as well as to increase the frequency of use of the hand and upper limb on the paralyzed side. As for hemiparesis of the upper limb, we provided repeated combined motion training of both hands using objects such as those used in the activity of daily living. As for the ataxia of the upper limb, we repeatedly provided enhancing training of peripheral sensory input (i.e., wrapping bandage), wearing a weight of $250 \mathrm{~g}$ on the wrist, and reaching movement aimed at improving intention tremor and dysmetria. A 60-min self-exercise was encouraged in another quiet room without any supervisors after intensive OT. The occupational therapist provided the self-exercise program that met the needs of the patient after discussing the program content with the patient.

\section{Outcome Measures}

The primary outcome was the Fugl-Meyer assessment (FMA) that was serially applied to evaluate functional motor changes in the affected upper limb.

The secondary outcomes were the modified Ashworth scale (MAS) and the International Cooperative Ataxia Rating Scale (ICARS). The severity of spasticity was evaluated using MAS. The severity of ataxia was evaluated by using the ICARS, that is a semi-quantitative rating scale that was proposed by the World Federation of Neurology. The ICARS total score is 0100 points and is based on all 19 items. The scale involves a compartmentalized quantification of postural and stance disorders, kinetic functions (limb ataxia), speech disorders (dysarthria), and oculomotor disorders.

All of these evaluations were performed on the day of admission and the day before discharge and were administered by an occupational therapist. Changes from admission to discharge were computed and compared using a Wilcoxon signed-rank test. A $p$ value $<0.05$ was considered to be statistically significant. All statistical analyses were performed using SPSS package 19.0 (SPSS, Chicago, IL, USA).

\section{Results}

The scheduled 15-day protocol of the intervention was completed by all 7 patients. None of the patients experienced any pathological symptoms or any deterioration of motor function in the upper limb during hospitalization and 4 weeks after discharge.

As shown in Table 2, the total score of the FMA improved significantly from admission to discharge. The MAS score did not significantly change for all the muscles (elbow flexors, wrist flexors, and finger flexors).

The total ICARS score decreased significantly. The scores of the postural and stance disorders component and the kinetic functions component (limb ataxia) improved significantly. In contrast, the scores of the speech disorders (dysarthria) component and the oculomotor disorders compartment did not significantly change.

\section{Discussion}

In the current study, all 7 patients with nonsensory $\mathrm{AH}$ due to thalamic a hemorrhage safely completed the NEURO-15 protocol (LF-rTMS and intensive OT). The protocol signifi- 


\section{Case Reports in Neurology}

cantly increased the FMA score and decreased the total ICARS score, suggesting improvement of motor function in the affected upper limb and the ipsilateral ataxia.

Fourteen AH cases were first reported by Fisher and Cole [1]. All but 1 patient had normal sensation. In several subsequent studies [4], AH was commonly defined as ipsilateral ataxia and hemiparesis without a sensory disturbance. Gorman et al. [9] reported that 45 of 1,468 lacunar infarctions (3\%) corresponded to $\mathrm{AH}$, and 10 of those cases $(0.7 \%)$ were due to a thalamic lesion. However, some patients with a sensory disturbance were also included. In addition, Dobato et al. [10] described 5 patients with incoordination and corticospinal tract signs in the limb opposite a dorsolateral thalamic hemorrhage. The incoordination of the patients of that study was reportedly due to a deep sensory loss, and thus the term "sensory $\mathrm{AH}^{\prime}$ was used to distinguish these patients from those with $\mathrm{AH}$. In the present study, we evaluated patients with only hemiparesis and ataxia, excluding patients with sensory loss.

A previous study reported that nonsensory AH due to a thalamic lesion is usually caused by lacunar infarctions [4]. The thalamus is a site of predilection not only for lacunar infarction but also for intracranial hemorrhage. Therefore, in the present study, we speculate that the lesion of the thalamic hemorrhage might involve the internal capsule near the thalamus. Few studies have investigated nonsensory AH due to a thalamic hemorrhage [5]; thus, the present report is a valuable resource.

An important pathway for the regulation of the motor functions of the cerebellum is the fronto-pontine-cerebellum-thalamus-sensorimotor cortical loop [11]. Cerebrocortical information enters the cerebellum at the middle cerebellar peduncle via the pontine nuclei and exercise feedback information enters the cerebellum at the lower cerebellar nucleus via the inferior olivary nucleus. In contrast, processed cerebellar information is transmitted to the primary motor cortex through the superior cerebellar peduncle via the thalamus. AH could be produced by lesions that are positioned anywhere within these loops. Kikuchi et al. [7] used the paired-pulse TMS technique to investigate the electrophysiological mechanisms of $\mathrm{AH}$ in patients whose responsible lesion was located in the posterior limb of the internal capsule and the thalamus.

Tanaka et al. [12] reported that 4 patients with $\mathrm{AH}$ due to thalamic lesions ( 3 due to a hemorrhage and 1 due to an infarction) showed crossed cerebellar diaschisis (CCD). This finding indicates that CCD occurs with ipsilateral ataxia and suggests that $\mathrm{AH}$ was not only the result of a disruption of the cortico-ponto-cerebellar pathway (i.e., the cerebellar afferent pathway) but also of the dentato-rubro-thalamic pathway (i.e., the cerebellar efferent pathway). In particular, CCD associated with hemiataxia in patients with thalamic lesions was assumed to result from a retrograde deactivation of the cerebellar hemisphere via the cerebellar efferent pathway.

All of the patients in the present study presented with hemiparesis, and we speculate that the lesional areas involve the internal capsule near the thalamus. This idea suggests that the $\mathrm{AH}$ in our patients resulted not only from a retrograde degeneration of the cerebellar efferent pathway but also from an anterograde degeneration of the cerebellar afferent pathway. However, the subjects in the present study were at the chronic disease stage. We did not acquire accurate information about the symptom course from the disease onset or about time-dependent changes in the brain CT scan. That is, the brain CT scans taken at admission to our hospital are insufficient to identify the responsible lesion and mechanism of the clinical symptoms of the patients. 


\section{Case Reports in Neurology}

Recently, rTMS has been introduced as a therapeutic tool for upper-limb hemiparesis after stroke. The probable efficacy of the LF-rTMS of contralesional motor cortex for chronic motor stroke was highlighted by the current evidence-based guideline [13]. In addition, we reported that the NEURO-15 protocol (LF-rTMS and intensive OT) significantly improved the motor function of the affected upper limb in post-stroke patients [6]. Furthermore, Yamada et al. [14] performed functional magnetic resonance imaging during repetitive flexion and extension movements of the finger before and after performing the NEURO-15 protocol. The results indicated that the intervention can induce functional cortical reorganization in Brodmann area 4 and 6, leading to functional motor recovery of the affected upper limb. Based on these findings, we postulated that the NEURO-15 protocol can produce further enhancement of brain plasticity and reorganization in the contralateral hemisphere and the cerebellum through the cerebellar afferent and efferent pathways, and thus better functional recovery of motor function. To our knowledge, the present investigation is the first to show a safe and feasible application of LF-rTMS and intensive OT for upper-limb hemiparesis and ataxia $(\mathrm{AH})$ without sensory disturbance that was due to a thalamic hemorrhage. The results of the present study suggest the possibility that the NEURO-15 protocol may be effective for patients with $\mathrm{AH}$ who significantly recovered from not only motor function of the affected upper limb but also from ipsilateral ataxia.

The training amount and environment, in addition to performing a substantial amount of task-oriented training, are all necessary to promote the functional reorganization of the brain for efficient and effective neurorehabilitation. In addition, functional magnetic resonance imaging demonstrated that intensive OT facilitated brain plasticity of the affected hemisphere [15]. In the present study, a substantial amount of task-oriented training was included in the exercise program. We speculate that, as a result of this exercise, a change occurred in the affected hemisphere that improved upper-limb function.

\section{Limitations}

Our study has limitations. First, we used the ICARS to evaluate ataxia in the present study. However, the ICARS may be not appropriate for the evaluation of AH patients because it is used to assess the need for pharmacotherapy for cerebellar ataxias such as spinocerebellar degeneration. The present study also showed a significant improvement in the ICARS score, and the FMA score was significantly improved as well. We cannot deny the possibility that the improvement of upper-limb hemiparesis influenced the improvement of the ICARS score. Second, the design of this study did not allow us to evaluate the separate influence of the intensive OT and the application of LF-rTMS. Further studies to assess this issue are needed. Third, we also could not examine the direct effect of the dentato-rubro-thalamic pathway and the status of contralateral cerebellar activation because electrophysiological evaluation and neuroimaging were not performed. Using previously published methods $[7$, $14]$, we intend to examine the mechanism of improvement in the future.

\section{Conclusions}

We used the NEURO-15 protocol (LF-rTMS and intensive OT) in 7 patients with nonsensory AH due to a thalamic hemorrhage. Our report firstly reveals the safety and feasibility of 


\section{Case Reports in Neurology} Case Rep Neurol 2017;9:179-187
\begin{tabular}{l|l}
\hline DOI: $10.1159 / 000478975$ & $\begin{array}{l}\text { C } 2017 \text { The Author(s). Published by S. Karger AG, Basel } \\
\text { www.karger.com/crn }\end{array}$
\end{tabular}

Urushidani et al.: Combination Treatment of LF-rTMS and Intensive OT for Ataxic Hemiparesis due to Thalamic Hemorrhage

the NEURO-15 intervention for upper-limb hemiparesis with ataxia. The results of the present study support the possibility that the NEURO-15 intervention may be beneficial not only for upper-limb hemiparesis but also for ipsilateral ataxia.

\section{Acknowledgements}

On behalf of the NEURO investigators of the Nishi-Hiroshima Rehabilitation Hospital, the authors gratefully acknowledge the support and participation of the patients in the present study. The NEURO investigators of the Nishi-Hiroshima Rehabilitation Hospital are Takatsugu Okamoto, MD, PhD; Eiji Inoue, OTR, MS; Sachiko Shiraoka, OTR; Naoki Urushidani, OTR; Hiroaki Tamashiro, OTR; Takashi Kurushima, OTR; Takashi Matsuda, OTR; Makoto Murakami, OTR; Chiho Oka, OTR; Nozomi Takagi, OTR; Shinya Osako, OTR; Yumiko Okuda, OTR; Takuya Ojimo, OTR; Haruna Okada, OTR; Haruka Ode, OTR; and Chihiro Murakami, OTR.

\section{Statement of Ethics}

The present study was carried out in compliance with the Declaration of Helsinki. The protocol of the study and intervention received the approval of the Ethics Committee of both Jikei University School of Medicine and Nishi-Hiroshima Rehabilitation Hospital, and informed consent was obtained from all subjects before entry into the study.

\section{Disclosure Statement}

The authors report no conflicts of interest. The authors alone are responsible for the content and writing of the present paper.

\section{References}

1 Fisher CM, Cole M: Homolateral ataxia and crural paresis: a vascularsyndrome. J Neurol Neurosurg Psychiatry 1965:28:48-55.

Fisher CM: Ataxic hemiparesis: a pathologic study. Arch Neurol 1978:35:126-128.

Boiten J, Lodder J: Ataxic hemiparesis following thalamic infarction. Stroke 1990;21:339-340.

Moulin T, Bogousslavsky J, Chopard JL, Ghika J, Crepin-Leblond T, Martin V, Maeder P: Vascular ataxic hemiparesis: a re-evaluation. J Neurol Neurosurg Psychiatry 1995;58:422-427.

5 van Blercom N, Manto M, Jacquy J, Hildebrand J: Dissociation in the neural control of single-joint and multi-joint movements in the thalamic ataxia syndrome. J Neurol Sci 1997;151:71-77.

-6 Kakuda W, Abo M, Shimizu M, Sasanuma J, Okamoto T, Yokoi A, Taguchi K, Mitani S, Harashima H, Urushidani N, Urashima M: A multi-center study on low-frequency rTMS combined with intensive occupational therapy for upper limb hemiparesis in post-stroke patients. J Neuroeng Rehabil 2012;9.4. Kikuchi S, Mochizuki H, Moriya A, Nakatani-Enomoto S, Nakamura K, Hanajima R, Ugawa Y. Ataxic hemiparesis: neurophysiological analysis by cerebellar transcranial magnetic stimulation. Cerebellum 2012;11:259-263.

-8 Wassermann EM: Risk and safety of repetitive transcranial magnetic stimulation: report and suggested guidelines from the International Workshop on the Safety of Repetitive Transcranial Magnetic Stimulation, June 5-7, 1996. Electroencephalogr Clin Neurophysiol 1998;108:1-16. 


\section{Case Reports in Neurology}

-9 Gorman MJ, Dafer R, Levine SR: Ataxic hemiparesis: critical appraisal of a lacunar syndrome. Stroke 1998;29:2549-2555.

10 Dobato JL, Villanueva JA, Gimenez-Roldan S: Sensory ataxic hemiparesis in thalamic hemorrhage. Stroke 1990;21:1749-1753.

11 Mochizuki H, Ugawa Y: Disappearance of essential tremor after stroke: which fiber of cerebellar loops is involved in posterior limb of the internal capsule? Mov Disord 2011;26:1577.

12 Tanaka M, Kondo S, Hirai S, Ishiguro K, Ishihara T, Morimatsu M: Crossed cerebellar diaschisis accompanied by hemiataxia: a PET study. J Neurol Neurosurg Psychiatry 1992;55:121-125.

13 Lefaucheur JP, André-Obadia N, Antal A, Ayache SS, Baeken C, Benninger DH, Cantello RM, Cincotta M, de Carvalho M, De Ridder D, Devanne H, Di Lazzaro V, Filipović SR, Hummel FC, Jääskeläinen SK, Kimiskidis VK, Koch G, Langguth B, Nyffeler T, Oliviero A, Padberg F, Poulet E, Rossi S, Rossini PM, Rothwell JC, Schönfeldt-Lecuona C, Siebner HR, Slotema CW, Stagg CJ, Valls-Sole J, Ziemann U, Paulus W, Garcia-Larrea L: Evidence-based guidelines on the therapeutic use of repetitive transcranial magnetic stimulation (rTMS). Clin Neurophysiol 2014;125:2150-206.

14 Yamada N, Kakuda W, Senoo A, Kondo T, Mitani S, Shimizu M, Abo M: Functional cortical reorganization after low-frequency repetitive transcranial magnetic stimulation plus intensive occupational therapy for upper limb hemiparesis: evaluation by functional magnetic resonance imaging in poststroke patients. Int J Stroke 2013;8:422-429.

15 Levy CE,Nichols DS, Schamalbrock PM, Keller P, Chakeres DW: Functional MRI evidence of cortical reorganization in upper limb stroke hemiplegia treated with constraint-induced movement therapy. Am J Phys Med Rehabil 2001;80:4-12.

\begin{tabular}{|c|c|c|c|c|c|c|c|}
\hline & Day 1 & Day 2-5 & Day 6 & Day $7-12$ & Day 13 & Day 14 & Day 15 \\
\hline & Tue & Wed - Sat & Sun & Mon-Sat & Sun & Mon & Tue \\
\hline \multirow{3}{*}{ Morning } & \multirow{3}{*}{ Admission } & $\begin{array}{l}\text { Low-frequency rTMS } \\
(20 \mathrm{~min})\end{array}$ & \multirow{6}{*}{ 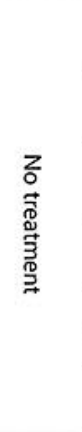 } & $\begin{array}{l}\text { Low-frequency rTMS } \\
(20 \mathrm{~min})\end{array}$ & \multirow{6}{*}{ 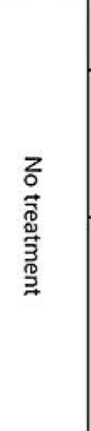 } & $\begin{array}{l}\text { Low-frequency rTMS } \\
\text { (20 min) }\end{array}$ & Interview \\
\hline & & $\begin{array}{l}\text { One-to-one training } \\
\text { (60 } \mathrm{min})\end{array}$ & & $\begin{array}{l}\text { One-to-one training } \\
(60 \mathrm{~min})\end{array}$ & & \multirow{2}{*}{$\begin{array}{l}\text { Self training } \\
\text { (120 min) }\end{array}$} & \multirow{2}{*}{ Discharge } \\
\hline & & $\begin{array}{l}\text { Self training } \\
\text { (60 min) }\end{array}$ & & $\begin{array}{l}\text { Self training } \\
(60 \mathrm{~min})\end{array}$ & & & \\
\hline \multirow{3}{*}{ Afternoon } & \multirow{3}{*}{$\begin{array}{l}\text { Pre-therapy } \\
\text { evaluation }\end{array}$} & $\begin{array}{c}\text { Low-frequency rTMS } \\
\text { (20 min) }\end{array}$ & & $\begin{array}{l}\text { Low-frequency rTMS } \\
(20 \mathrm{~min})\end{array}$ & & \multirow{3}{*}{$\begin{array}{l}\text { Post therapy } \\
\text { evaluation }\end{array}$} & \\
\hline & & $\begin{array}{l}\text { One-to-one training } \\
\text { (60 } \mathrm{min})\end{array}$ & & $\begin{array}{l}\text { One-to-one training } \\
\text { (60 min) }\end{array}$ & & & \\
\hline & & $\begin{array}{l}\text { Self training } \\
\text { (60 min) }\end{array}$ & & $\begin{array}{l}\text { Self training } \\
(60 \mathrm{~min})\end{array}$ & & & \\
\hline
\end{tabular}

Fig. 1. Schedule of the NEURO-15 protocol. 


\section{Case Reports in Neurology}

Table 1. Clinical characteristics of the studied patients

\begin{tabular}{|c|c|c|c|c|c|c|c|}
\hline Age, years & 58 & 77 & 62 & 63 & 74 & 67 & 73 \\
\hline Sex & female & female & male & female & male & female & male \\
\hline Diagnosis & $\mathrm{TH}$ & $\mathrm{TH}$ & $\mathrm{TH}$ & TH & $\mathrm{TH}$ & $\mathrm{TH}$ & $\mathrm{TH}$ \\
\hline Handedness & right & right & right & right & right & right & right \\
\hline Affected side & right & left & right & left & right & right & left \\
\hline MMSE score & 29 & 24 & 28 & 30 & 26 & 29 & 29 \\
\hline $\begin{array}{l}\text { Time after onset of stroke, months } \\
\text { Brunnstrom stage }\end{array}$ & 96 & 146 & 30 & 78 & 43 & 60 & 13 \\
\hline (hand-fingers/upper limb) & $5 / 5$ & $5 / 5$ & $5 / 5$ & $5 / 5$ & $5 / 5$ & $5 / 5$ & $5 / 5$ \\
\hline Upper-limb sensation & & & & & & & \\
\hline (SIAS light touch/position) & $3 / 3$ & $3 / 3$ & $3 / 3$ & $3 / 3$ & $3 / 3$ & $3 / 3$ & $3 / 3$ \\
\hline Dysarthria & + & - & - & - & - & + & - \\
\hline
\end{tabular}

TH, thalamic hemorrhage; MMSE, Mini-Mental State Examination; SIAS, Stroke Impairment Assessment Set. (+) indicates that a deficit is present and (-) indicates the absence of a deficit.

Table 2. Changes in test scores after performing the NEURO-15 protocol

\begin{tabular}{lccc}
\hline & At admission & At discharge & $p$ value \\
\hline$F M A$ & & & $0.043^{*}$ \\
Total score & $54.9 \pm 8.5$ & $56.7 \pm 9.5$ & $\mathrm{~ns}$ \\
Score for category A & $34.3 \pm 1.9$ & $34.3 \pm 1.9$ & $\mathrm{~ns}$ \\
Score for category B & $9.3 \pm 0.8$ & $9.7 \pm 0.5$ & $0.043^{*}$ \\
Score for category C & $13.4 \pm 0.8$ & $13.9 \pm 0.4$ & $0.018^{*}$ \\
Score for category D & $0.9 \pm 0.7$ & $2.3 \pm 1.1$ & \\
\hline ICARS & & & $0.028^{*}$ \\
Total score & $26.6 \pm 11.1$ & $21.0 \pm 10.2$ & $0.028^{*}$ \\
Score for postural and stance disorders & $11.4 \pm 7.7$ & $9.3 \pm 7.4$ & $0.028^{*}$ \\
Score for kinetic functions & $14.4 \pm 3.6$ & $11.0 \pm 2.5$ & $\mathrm{~ns}$ \\
Score for speech disorders & $1.0 \pm 1.5$ & $1.0 \pm 1.5$ & $\mathrm{~ns}$ \\
Score for oculomotor disorders & $0.1 \pm 0.3$ & $0.0 \pm 0.0$ & $\mathrm{~ns}$ \\
\hline$M A S$ & & & $\mathrm{~ns}$ \\
Elbow flexors & $0.3 \pm 0.4$ & $0.0 \pm 0.0$ & $\mathrm{~ns}$ \\
Wrist flexors & $0.1 \pm 0.3$ & $0.1 \pm 0.3$ & $0.0 \pm 0.0$ \\
Finger flexors & $0.3 \pm 0.5$ & & \\
\hline
\end{tabular}

FMA, Fugl-Meyer assessment; ICARS, International Cooperative Ataxia Rating scale; MAS, modified Ashworth scale. ${ }^{*} p<0.05$. 